\title{
Application of Singular Perturbation Methods for Three-Dimensional Minimum-Time Interception
}

\author{
Donglong Sheu* \\ National Cheng-Kung University, Tainan, Taiwan, Republic of China \\ and \\ Nguyen X. Vinh $\dagger$ and Robert M. Howe‡ \\ University of Michigan, Ann Arbor, Michigan 48109
}

\begin{abstract}
In this paper, a feedback control law based on the singular perturbation method is developed for three-dimensional minimum-time interception. Whereas the heading and flight-path angles are considered fast variables with the same time scale, the relative position and the specific energy are considered slow variables. A zeroth-order optimal control algorithm is developed, and an extension to higher-order analysis is discussed. With the demonstrations of several numerical examples, it is shown that this time-scale separation is physically reasonable and results in a uniformly valid control law for long-, medium-, and short-range interception.
\end{abstract}

\section{Nomenclature}

$A, a, a_{i r}, a_{0 f}=$ parameters defined in Eqs. (40), (54), (B8), and (B12), respectively

$B, b, b_{i r}, b_{0 f}=$ parameters defined in Eqs. (41), (55), (B9), and (B13), respectively

$B_{R} \quad=$ parameter defined in Eq. (52)

$C_{D_{0}} \quad=$ zero-lift drag coefficient

$C_{L} \quad=$ lift coefficient

$D \quad=$ drag force

$D_{i} \quad=$ induced drag in rectilinear level flight

$D_{0} \quad=$ zero-lift drag force

$d_{f} \quad=$ capture radius

$E \quad=$ specific energy of the interceptor

$E_{r} \quad=$ the ratio $\mathrm{d} \gamma^{i} / \mathrm{d} \psi^{i}$ evaluated at $\tau=\tau_{0}$

e $\quad=$ unit tangent vector, $\cos \gamma \cos \psi i+\cos \gamma$ $\sin \psi \boldsymbol{j}-\sin \gamma \boldsymbol{k}$

$f \quad=$ dynamic function (with subscript)

$f \quad=$ vector of dynamic functions (with subscript)

$g=$ acceleration of gravity

$g_{i} \quad=$ quantities defined in Eqs. (A22-A25)

$H \quad=$ Hamiltonian

$h, h_{T} \quad=$ interceptor's altitude and target's altitude, respectively

$I \quad=$ integral (with subscript)

$i, j, k=$ unit vectors in inertial frame $O X Y Z$ (see Fig. 1)

$J \quad=$ payoff function

$K \quad=$ induced-drag parameter

$L \quad=$ lift force

$M \quad=$ Mach number

$n, n_{c}, n_{s}=$ aerodynamic load factor, $n \cos \phi$ and $n \sin \phi$, respectively

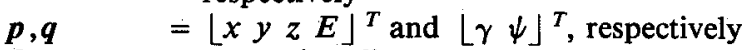

$R \quad=$ separation distance

$\mathbf{R} \quad=$ universal gas constant

$\boldsymbol{R} \quad=\boldsymbol{R}_{I}-\boldsymbol{R}_{T}$

Received Sept. 16, 1988; revision received Jan. 29, 1990. Copyright (C) 1990 by the American Institute of Aeronautics and Astronautics, Inc. All rights reserved.

*Instructor, Department of Aeronautical and Astronautical Engineering; currently, Graduate Student, Department of Aerospace Engineering, The University of Michigan. Student Member AIAA.

†Professor, Department of Aerospace Engineering. Member AIAA. $\ddagger$ Professor, Department of Aerospace Engineering. Associate Fellow AIAA.
$\boldsymbol{R}_{I} \quad=$ interceptor's position vector in inertial frame OXYZ (see Fig. 1)

$\boldsymbol{R}_{T} \quad=$ target's position vector in inertial frame $O X Y Z$ (see Fig. 1)

$S \quad=$ interceptor's wing area

$T, \mathrm{~T} \quad=$ thrust and temperature (Table 1 ), respectively

$t \quad=$ time

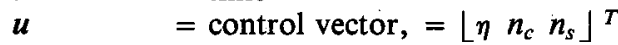

$u_{T}, v_{T}, w_{T}=$ target's velocity components

$\boldsymbol{V}, \boldsymbol{V}=$ interceptor's velocity and velocity vector, respectively

$V_{E}, V_{h} \quad=\partial V / \partial E$ and $\partial V / \partial h$, respectively

$V_{T}, V_{T} \quad=$ target's velocity and velocity vector, respectively

$W \quad=$ interceptor's weight

$X, Y, Z \quad=$ inertial frame coordinates (see Fig. 1)

$x, y, z \quad=$ components of vector $\boldsymbol{R}$

$\gamma \quad=$ interceptor's flight-path angle

$\epsilon \quad=$ artificial perturbation parameter

$\eta \quad=$ throttle control

$\eta_{k} \quad=$ proportional constant defined in Eq. (A32)

$\boldsymbol{\Lambda}=$ vector of adjoint variables (with subscript)

$\lambda=$ adjoint variable (with subscript)

$\nu \quad=$ constant multiplier defined in Eqs. (19-21)

$\rho \quad=$ air density

$\sigma_{E} \quad=$ proportional constant defined in Eqs. (B10) and (B11)

$\tau=$ stretched time for inner expansions,

$=\tau_{0}+\left(t-t_{0}\right) / \epsilon$

$=\left(x^{2}+y^{2}+z^{2}-d_{f}^{2}\right) / 2$

$=$ interceptor's bank angle

= interceptor's azimuth (heading) angle

Superscripts

$i=$ inner expansion

o $\quad=$ outer expansion

$r=$ reduced order

(i) = differentiation with time

Subscripts

$A \quad=$ average

$f \quad=$ final value

I $\quad$ interceptor

(k) $\quad=$ term corrected to the $k$ th order

$T \quad=$ target

$0 \quad$ = initial value, also zeroth-order term 


\section{Introduction}

$\mathbf{F}$ OR nearly two decades, the singular perturbation method has been highlighted in solving various optimal trajectory problems. For example, Calise ${ }^{1}$ discussed the application of this method for variational problems in aircraft flight. $\mathrm{Ar}$ dema $^{2}$ used this method with matched asymptotic expansions to solve the minimum time-to-climb problem. Shridhar and Gupta ${ }^{3}$ derived missile guidance laws with the assumption that the gravity is negligible in comparison with both the missile and target acceleration capabilities. Recently, Visser and Shinar ${ }^{4}$ carried out an analysis of the horizontal plane interception problem based on a forced singular perturbation method.

In the cases solved in Refs. 3 and 4 , either the gravity is neglected or the flight is assumed to be in a horizontal plane; thus, the problems are greatly simplified. The extension of the analysis to include the gravity effect in three-dimensional interception problems is a little more involved. Successful analyses for these problems, including the gravity effect, were made by the energy formulation with suitable time-scale assumptions. ${ }^{5-12}$ For example, Calise ${ }^{11}$ analyzed a three-dimensional interception problem, using the energy formulation and assuming the altitude to be a faster variable in comparison with the horizontal range components. In that case, the zerothorder approximation leads to an essentially horizontal problem. Since the altitude rate is neglected, range matching is adopted to arrive at the target altitude when the separation in the horizontal plane is driven to zero. Do Khac and Huynh ${ }^{12}$ used similar approaches and compared the results with the "exact" open-loop optimal solutions based on the gradient method. It is shown that a better than $99 \%$ accuracy is achieved for the performance index.

In this study of three-dimensional minimum-time interception, if the vertical separation $z$ between the interceptor and the target and the specific energy $E$ are scaled as fast variables in comparison with the horizontal distances, in longitude $x$ and latitude $y$ (as has been done in Refs. 11 and 12), the resulting control laws are valid only when $x$ and $y$ are much larger than $z$ and for a speed $V$ in a range near to its maximum value $V_{\max }$. In other words, this time-scale separation is most suitable for the case of near-horizontal interception starting with a high initial speed. With this limitation in mind, we assume in this paper that $x, y, z$, and $E$ are slow variables, while only the flight-path angle $\gamma$ and the heading angle $\psi$ are fast variables with the same time scale. This assumption is physically reasonable since $z$ and $E$ cannot increase very rapidly, and the turning rates $\dot{\gamma}$ and $\dot{\psi}$ should be on approximately the same time scale in order to uniformly rotate the velocity from one direction to another. The resulting algorithm is more involved than those of Refs. 11 and 12, and consequently is more time consuming. As such, an effort has been made to reduce the computation time and to accelerate the convergence of the solution as will be shown in the text.

The derivations of the equations of motion and the necessary conditions for optimality are based on Refs. 13-15. Then the zeroth-order singular perturbation analysis based on the present assumption is carried out. Numerical examples, using basic aircraft parameters from Ref. 16, are given to demonstrate the effectiveness of the method. Closed-loop simulations are conducted for various interception conditions. The essential features of the optimal trajectories are discussed. To evaluate the accuracy of the zeroth-order solution, a recursive algorithm for higher-order analysis, briefly described is Sec. V, is examined for one numerical case.

\section{Formulation of Three-Dimensional Interception}

With the assumption of a constant-mass interceptor and a fixed flat Earth, the equations of motion for three-dimensional interception are formulated as

$$
\dot{x}=f_{x}=V \cos \gamma \cos \psi-u_{T}, \quad x\left(t_{0}\right)=x_{0}
$$

$$
\begin{aligned}
\dot{y}=f_{y}=V \cos \gamma \sin \psi-v_{T}, & y\left(t_{0}\right)=y_{0} \\
\dot{z}=f_{z}=-V \sin \gamma-w_{T}, & z\left(t_{0}\right)=z_{0} \\
\dot{E}=f_{E}=[(T-D) V] / W, & E\left(t_{0}\right)=E_{0} \\
\dot{\gamma}=f_{\gamma}=(g / V)\left(n_{c}-\cos \gamma\right), & \gamma\left(t_{0}\right)=\gamma_{0} \\
\dot{\psi}=f_{\psi}=(g / V)\left(n_{s} / \cos \gamma\right), & \psi\left(t_{0}\right)=\psi_{0}
\end{aligned}
$$

where $x, y$, and $z$ are the separation distance components of the vector $\boldsymbol{R}=\boldsymbol{R}_{I}-\boldsymbol{R}_{T}$, with the $z$ direction along the gravity acceleration. Figure 1 shows this geometry at the initial time $t_{0}$. Note that $O X Y Z$ is an inertial frame. These equations are valid for a point mass interceptor with the thrust $T$ directed along the tangent to the flight path. It is commonly assumed that the thrust $T$, drag $D$, and lift $L$ can be modeled as follows:

$$
\begin{gathered}
T=\eta T_{\max }(h, M) \\
D=D_{0}(h, V)+D_{i}(h, V)\left(n_{c}^{2}+n_{s}^{2}\right) \\
D_{0}(h, V)=1 / 2 \rho(h) V^{2} S C_{D_{0}}(M) \\
D_{i}(h, V)=K(M) W^{2} /\left[1 / 2 \rho(h) V^{2} S\right] \\
L=n W
\end{gathered}
$$

where the throttle control $\eta$ and the load factor $n$ are subject to the following constraints:

$$
\begin{gathered}
\eta_{\min }(h, M) \leq \eta \leq 1 \\
n \leq n_{\max }
\end{gathered}
$$

The maximum load factor $n_{\max }$ is constrained by the maximum structural load or the maximum aerodynamic load, whichever is smaller. The energy state $E$ is related to $h$ and $V$ by

$$
E=h+\left(V^{2} / 2 g\right)
$$

and the heading and flight-path controls $n_{s}$ and $n_{c}$ are related to the load factor $n$ and the bank angle $\phi$ by

$$
\begin{aligned}
& n_{c}=n \cos \phi \\
& n_{s}=n \sin \phi
\end{aligned}
$$

where $-\pi<\phi \leq \pi$

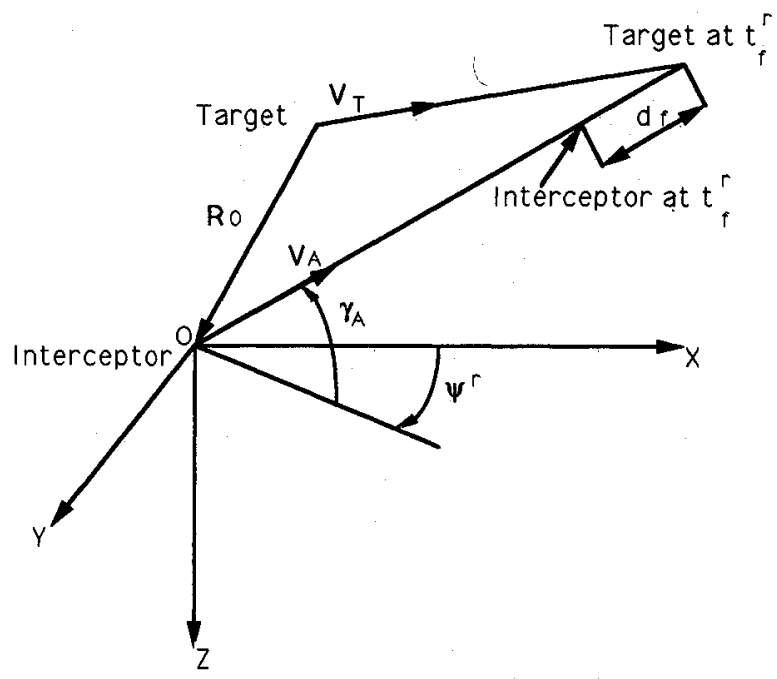

Fig. 1 Geometry of interception at initial time. 


\section{Optimal Control Formulation}

The problem of interest is to find a set of controls $\eta(t)$, $n_{c}(t)$, and $n_{s}(t)$ that leads to successful interception and minimizes

$$
J=\int_{0}^{t_{f}} \mathrm{~d} t
$$

subject to the constraints in Eqs. (10) and (11). Successful interception (capture) is determined by the conditions

$$
\begin{gathered}
\mathbf{R}\left(t_{f}\right)=\sqrt{x^{2}+y^{2}+z^{2}}=d_{f} \\
\dot{\mathbf{R}}\left(t_{f}\right)<0
\end{gathered}
$$

where $d_{f}$ is the "capture radius" representing the interceptor's firing envelope. ${ }^{4}$

The Hamiltonian of the system is

$$
\begin{aligned}
H & =1+\lambda_{x} f_{x}+\lambda_{y} f_{y}+\lambda_{z} f_{z}+\lambda_{E} f_{E}+\lambda_{\gamma} f_{\gamma}+\lambda_{\psi} f_{\psi} \\
& + \text { constraints }
\end{aligned}
$$

Consequently, the Euler-Lagrange equations and the transversality conditions are

$$
\begin{aligned}
& \dot{\lambda}_{x}=-\frac{\partial H}{\partial x}=0, \quad \lambda_{x}\left(t_{f}\right)=\nu x_{f} \\
& \dot{\lambda}_{y}=-\frac{\partial H}{\partial y}=0, \quad \lambda_{y}\left(t_{f}\right)=\nu y_{f} \\
& \dot{\lambda}_{z}=-\frac{\partial H}{\partial z}=\frac{\partial H}{\partial h} \\
& =\lambda_{x} V_{h} \cos \gamma \cos \psi+\lambda_{y} V_{h} \cos \gamma \sin \psi-\lambda_{z} V_{h} \sin \gamma \text { : } \\
& +\lambda_{E} \frac{\partial}{\partial h}\left[\frac{(T-D) V}{W}\right]-\lambda_{\gamma} V_{h} \frac{g}{V^{2}}\left(n_{c}-\cos \gamma\right) \\
& -\lambda_{\psi} V_{h} \frac{g}{V^{2}} \frac{n_{s}}{\cos \gamma}+\frac{\partial}{\partial h} \text { (constraints), } \quad \lambda_{z}\left(t_{f}\right)=\nu z_{f} \\
& \dot{\lambda}_{E}=-\frac{\partial H}{\partial E} \\
& =-\lambda_{x} V_{E} \cos \gamma \cos \psi-\lambda_{y} V_{E} \cos \gamma \sin \psi+\lambda_{z} V_{E} \sin \gamma \\
& -\lambda_{E} \frac{\partial}{\partial E}\left[\frac{(T-D) V}{W}\right]+\lambda_{\gamma} V_{E} \frac{g}{V^{2}}\left(n_{c}-\cos \gamma\right) \\
& +\lambda_{\psi} V_{E} \frac{g}{V^{2}} \frac{n_{s}}{\cos \gamma}-\frac{\partial}{\partial E} \text { (constraints), } \quad \lambda_{E}\left(t_{f}\right)=0 \\
& \dot{\lambda}_{\gamma}=-\frac{\partial H}{\partial \gamma} \\
& =\lambda_{x} V \sin \gamma \cos \psi+\lambda_{y} V \sin \gamma \sin \psi+\lambda_{z} V \cos \gamma \\
& -\lambda_{\gamma} \frac{g}{V} \sin \gamma-\lambda_{\psi} \frac{g}{V} \frac{n_{s} \sin \gamma}{\cos ^{2} \gamma}, \quad \lambda_{\gamma}\left(t_{f}\right)=0 \\
& \dot{\lambda}_{\psi}=-\frac{\partial H}{\partial \psi} \\
& =\lambda_{x} V \cos \gamma \sin \psi-\lambda_{y} V \cos \gamma \cos \psi, \quad \lambda_{\psi}\left(t_{f}\right)=0
\end{aligned}
$$

where $V_{h} \triangleq \partial V / \partial h$ and $V_{E} \triangleq \partial V / \partial E$.

The optimal throttle control $\eta_{\text {opt }}$ can be determined by minimizing

$$
H=1+\cdots+\lambda_{E} \frac{\eta T_{\max } V}{W}+\cdots
$$

with respect to $\eta$ subject to the constraint of Eq. (10). From the preceding equation, it is obvious that

$$
\eta_{\mathrm{opt}}= \begin{cases}\eta_{\min }(h, M), & \text { if } \lambda_{E}>0 \\ \text { variable, } & \text { if } \lambda_{E}=0 \\ 1, & \text { if } \lambda_{E}<0\end{cases}
$$

Similarly, the optimal controls $n_{c(\mathrm{opt})}$ and $n_{s(\mathrm{opt})}$ can be determined by minimizing $H$ with respect to $n_{c}$ and $n_{s}$. Accordingly,

$$
\begin{aligned}
& H_{n_{c}}=-2 \lambda_{E} V \frac{D_{i}(h, V) n_{c(\mathrm{opt})}}{W}+\lambda_{\gamma} \frac{g}{V}=0 \\
& H_{n_{s}}=-2 \lambda_{E} V \frac{D_{i}(h, V) n_{S(\mathrm{opt})}}{W}+\lambda_{\psi} \frac{g}{V} \frac{1}{\cos \gamma}=0
\end{aligned}
$$

which are adequate in case that $n_{c}^{2}+n_{s}^{2}<n_{\max }^{2}$.

Because the target velocity can be considered a constant for a sampled-data system the Hamiltonian $H$ is not explicitly dependent on the time and the necessary conditions for optimality lead to $\dot{H}=0$, or $H=$ const. Furthermore, because the terminal time $t_{f}$ is left open and the terminal condition as given by Eq. (16) is not explicitly dependent on the time, in this pursuit problem, it is necessary that $H_{f}=0$. Accordingly,

$$
H=0
$$

when the necessary conditions for optimality are satisfied.

\section{Zeroth-Order Singular Perturbation Analysis}

In this paper, we consider the relative position and the specific energy as slow variables and the heading and flight-path angles as fast variables. The outer-layer equations are

$$
\begin{array}{rlrl}
\frac{\mathrm{d} p^{o}}{\mathrm{~d} t} & =f_{p}\left(p^{o}, q^{o}, u^{o}\right), & & p^{o}\left(t_{0}\right)=p_{0} \\
\epsilon \frac{\mathrm{d} q^{o}}{\mathrm{~d} t} & =f_{q}\left(p^{o}, q^{o}, u^{o}\right) & & \\
\frac{\mathrm{d} \Lambda_{p}^{o}}{\mathrm{~d} t} & =-\frac{\partial H^{o}}{\partial p^{o}}, & \Lambda_{p}^{o}\left(t_{f}\right)=\left.\nu \frac{\partial \Phi}{\partial p}\right|_{p_{f}} \\
\epsilon \frac{\mathrm{d} \Lambda_{p}^{o}}{\mathrm{~d} t}=-\frac{\partial H^{o}}{\partial q^{o}}, & \Lambda_{q}^{o}\left(t_{f}\right)=0
\end{array}
$$

where $\left({ }^{\circ}\right)^{o}$ denotes the outer-layer variable and

$$
\begin{aligned}
& p \triangleq\left\lfloor\begin{array}{llll}
x & y & z & E
\end{array}\right\rfloor^{T}, \quad q \triangleq\left\lfloor\begin{array}{lll}
\gamma & \psi
\end{array}\right\rfloor^{T,} \quad u \triangleq\left\lfloor\begin{array}{lll}
\eta & n_{c} & n_{s}
\end{array}\right\rfloor^{T} \\
& f_{p} \triangleq\left\lfloor f_{x} f_{y} f_{z} f_{E}\right\rfloor^{T}, \quad f_{q} \triangleq\left\lfloor f_{\gamma} f_{\psi}\right\rfloor^{T} \\
& \Lambda_{p} \triangleq\left\lfloor\begin{array}{llll}
\lambda_{x} & \lambda_{y} & \lambda_{z} & \lambda_{E}
\end{array}\right\rfloor^{T}, \quad \Lambda_{q} \triangleq\left\lfloor\begin{array}{lll}
\lambda_{\gamma} & \lambda_{\psi}
\end{array}\right\rfloor^{T}
\end{aligned}
$$

To find the governing equations for the inner-layer variables, we use a stretched time scale $\tau=\tau_{0}+\left(t-t_{0}\right) / \epsilon$. The inner-layer equations can be represented as

$$
\begin{array}{cc}
\frac{\mathrm{d} p^{i}}{\mathrm{~d} \tau}=\epsilon f_{p}\left(p^{i}, q^{i}, u^{i}\right), & p^{i}\left(\tau_{0}\right)=p_{0} \\
\frac{\mathrm{d} q^{i}}{\mathrm{~d} \tau}=f_{q}\left(p^{i}, q^{i}, u^{i}\right), & q^{i}\left(\tau_{0}\right)=q_{0} \\
\frac{\mathrm{d} \Lambda_{p}^{i}}{\mathrm{~d} \tau}=-\epsilon \frac{\partial H^{i}}{\partial p^{i}} & \frac{\mathrm{d} \Lambda_{q}^{i}}{\mathrm{~d} \tau}=-\frac{\partial H^{i}}{\partial q^{i}}
\end{array}
$$

where ()$^{i}$ denotes the inner-layer variable. 


\section{Outer-Layer Analysis}

To find the zeroth-order outer-layer solution, we let $\epsilon=0$ in Eqs. (30) and (32). The solution to this problem is called the reduced-order solution and is denoted by superscript $r$. The problem is to find the optimal controls $\eta^{r}(t), \gamma^{r}(t)$, and $\psi^{\prime}(t)$ such that Eqs. (29-32) and the capture condition $\left[x^{r}\left(t_{f}^{r}\right)\right]^{2}+\left[y^{r}\left(t_{f}^{r}\right)\right]^{2}+\left[z^{r}\left(t_{f}^{r}\right)\right]^{2}=d_{f}^{2}$ are satisfied and $t_{f}^{r}$ is minimized. The solutions can be determined by guessing the four quantities $\gamma_{f}^{r}, \psi_{f}^{r}, t_{f}^{r}$, and $E_{f}^{r}$ and using the algorithm described in Appendix A to improve.

A good approach to guess $\gamma_{f}^{r}, \psi^{r}, t_{f}^{r}$, and $E_{f}^{r}$ is from physical consideration. As shown in Fig. 1, we assume $\gamma_{A}$ to be constant and guess the average velocity $V_{A}\left(V_{0} \leq V_{A} \leq V_{\max }\right)$ to solve the collision course equation

$$
-d_{f} \boldsymbol{e}_{A}=\boldsymbol{R}_{0}+\left(V_{A} \boldsymbol{e}_{A}-\boldsymbol{V}_{T}\right)\left(t_{f}^{r}-t_{0}\right)
$$

from which we obtain

$$
\begin{gathered}
t_{f}^{r}-t_{0}=1 /\left(A+\sqrt{A^{2}+B}\right) \\
\boldsymbol{e}_{A}=\frac{-\boldsymbol{R}_{0}+V_{T}\left(t_{f}^{r}-t_{0}\right)}{V_{A}\left(t_{f}^{r}-t_{0}\right)+d_{f}}
\end{gathered}
$$

where

$$
\begin{gathered}
A=\frac{R_{0} \cdot V_{T}+V_{A} d_{f}}{R_{0} \cdot R_{0}-d_{f}^{2}} \\
B=\frac{V_{A}^{2}-V_{T} \cdot V_{T}}{R_{0} \cdot R_{0}-d_{f}^{2}} \\
\boldsymbol{e}_{A} \triangleq \frac{V_{A}}{V_{A}}=\cos \gamma_{A} \cos \psi^{r} \boldsymbol{i}+\cos \gamma_{A} \sin \psi^{r} \boldsymbol{j}-\sin \gamma_{A} \boldsymbol{k}
\end{gathered}
$$

Then we let $\gamma_{f}^{r}=\gamma_{A}+\delta \gamma_{f}, V_{f}^{r}=2 V_{A}-V_{0}, h_{f}^{r}=h_{T}\left(t_{0}\right)+V_{T}\left(t_{f}^{r}\right.$ $\left.-t_{0}\right) \sin \gamma_{T}-d_{f} \sin \gamma_{f}^{r}$, and $E_{f}^{r}=h_{f}^{r}+\left(V_{f}^{r}\right)^{2} / 2 g$, where $\delta \gamma_{f}$ can be chosen to be 10-15 deg for a wide range of altitude separation.

Because all the variables vary slowly, the time increment for numerical integration of the slow modes alone can be much larger than that for simulation including both the slow and the fast modes. Following the procedure in Appendix A, we find that all the necessary conditions for optimality are satisfied except that the initial conditions are not matched. By assuming that the increments $\Delta \gamma_{f}^{r}, \Delta \psi^{r}, \Delta t_{f}^{r}$, and $\Delta E_{f}^{r}$ are certain linear functions of the differences between the specified and the computed initial conditions, we can improve $\gamma_{f}^{r}, \psi^{r}, t_{f}^{r}$, and $E_{f}^{r}$, correspondingly. Typically, four to six iterations are adequate.

\section{Inner-Layer Analysis}

Having obtained the reduced-order solution, we are in a position to find the inner-layer solution. By setting $\epsilon=0$ in Eqs. (33) and (35), the problem is then to find the optimal controls $n_{c}^{i}$ and $n_{s}^{i}$ such that Eqs. (33-36) and the final conditions $\gamma^{i}\left(\tau_{f}\right)=\gamma^{r}\left(t_{0}\right)$ and $\psi^{i}\left(\tau_{f}\right)=\psi^{r}$ are satisfied and the stretched final time $\tau_{f}$ is minimized. In this problem, the initial conditions $\lambda_{\gamma}^{i}\left(\tau_{0}\right)$ and $\lambda_{\psi}^{i}\left(\tau_{0}\right)$ are unknown. Instead of guessing both of them directly, it is better to guess the initial ratio $\mathrm{d} \gamma^{i} / \mathrm{d} \psi^{i}$ at $\tau=\tau_{0}$ as described in Eq. (53). Then from Eqs. (26-28) and (53), we can solve for $n_{c}^{i}, n_{s}^{i}, \lambda_{\gamma}^{i}$, and $\lambda_{\psi}^{i}$ simultaneously.

Recall from Appendix A the reduced-order solution that satisfies

$$
\begin{aligned}
H^{r} & =1+\Lambda_{0} \cdot\left(V_{0}^{r} e_{0}^{r}-V_{T}\right) \\
& +\lambda_{E 0}^{r} \frac{\left(\eta^{r} T_{\max }-D_{0}^{r}-D_{i}^{r} \cos ^{2} \gamma_{0}^{r}\right) V_{0}^{r}}{W}=0
\end{aligned}
$$

and the optimal control conditions

$$
\Lambda_{0} \cdot \frac{\partial e_{0}^{r}}{\partial \gamma_{0}^{r}}+\lambda_{E 0}^{r} \frac{D_{i}^{r} \sin 2 \gamma_{0}^{r}}{W}=0
$$

$$
\Lambda_{0} \cdot \frac{\partial e_{0}^{r}}{\partial \psi^{r}}=0
$$

where

$$
\Lambda_{0} \triangleq \lambda_{x}^{r} i+\lambda_{y}^{r} j+\lambda_{z 0}^{r} k
$$

Since $e_{0}^{r}, \partial e_{0}^{r} / \partial \gamma_{0}^{r}$, and $\partial e_{0}^{r} / \partial \psi$ are three mutually orthogonal unit vectors, we can define

$$
\mathbf{\Lambda}_{0}=\lambda_{1} \boldsymbol{e}_{0}^{r}+\lambda_{2} \frac{\partial e_{0}^{r}}{\partial \gamma_{0}^{r}}+\lambda_{3} \frac{\partial e_{0}^{r}}{\partial \psi^{r}}
$$

By substituting Eq. (46) into Eqs. (43-45) and solving for $\lambda_{1}$, $\lambda_{2}$, and $\lambda_{3}$, we obtain

$$
\begin{gathered}
\lambda_{3}=0 \\
\lambda_{2}=-\lambda_{E 0}^{r} \frac{D_{i}^{r} \sin 2 \gamma_{0}^{r}}{W}
\end{gathered}
$$

$$
\begin{aligned}
& \lambda_{1}= \\
& -\frac{1+\lambda_{E 0}^{r}\left[\left(\eta^{r} T_{\max }-D_{0}^{r}-D_{i}^{r} \cos ^{2} \gamma_{0}^{r}\right) V_{0}^{r} / W\right]-\lambda_{2}\left(V_{T} \cdot \partial e_{0}^{r} / \partial \gamma_{0}^{r}\right)}{V_{0}^{r}-V_{T} \cdot e_{0}^{r}}
\end{aligned}
$$

Theoretically, $V_{0}^{r}$ should be equal to $V_{0}$. However, since $\gamma_{0}^{r}, \psi^{r}$, and $\lambda_{E 0}^{r}$ are computed numerically, it is consistent to adopt the integrated value $V_{0}^{r}$ instead of the specified value $V_{0}$ in Eqs. (48) and (49).

From Eqs. (33) and (35), we find that $p^{i}(\tau)=p^{i}\left(\tau_{0}\right)$ and $\Lambda_{p}^{i}(\tau)=\Lambda_{p}^{i}\left(\tau_{0}\right)$ in the case that $\epsilon=0$. Then from the matching conditions $\boldsymbol{p}^{i}\left(\tau_{0}\right)=\boldsymbol{p}^{r}\left(t_{0}\right)$ and $\boldsymbol{\Lambda}_{p}^{i}\left(\tau_{0}\right)=\boldsymbol{\Lambda}_{p}^{r}\left(t_{0}\right)$, we obtain $p^{i}(\tau)=p^{r}\left(t_{0}\right)$ and $\boldsymbol{\Lambda}_{p}^{i}(\tau)=\boldsymbol{\Lambda}_{p}^{r}\left(t_{0}\right)$. Therefore, the Hamiltonian of the inner layer can be expressed as

$$
\begin{aligned}
H^{i} & =1+\Lambda_{0} \cdot\left(V_{0}^{r} e^{i}-V_{T}\right) \lambda_{E 0}^{r} V_{0}^{r} \\
& +\frac{\left\{\eta^{r} T_{\max }-D_{0}^{r}-D_{i}^{r}\left[\left(n_{c}^{i}\right)^{2}+\left(n_{s}^{i}\right)^{2}\right]\right\}}{W} \\
& +\lambda_{\gamma}^{i} \frac{g}{V_{0}^{r}}\left(n_{c}^{i}-\cos \gamma\right)+\lambda_{\psi}^{i} \frac{g}{V_{0}^{r}} \frac{n_{s}^{i}}{\cos \gamma^{i}}
\end{aligned}
$$

By eliminating $\lambda_{\gamma}^{i}$ and $\lambda_{\psi}^{i}$ in Eq. (50) using Eqs. (26) and (27) and then subtracting Eq. (43) from it, we obtain with the aid of Eqs. (46-49)

$$
\left(n_{\mathrm{c}}^{i}-\cos \gamma^{i}\right)^{2}+\left(n_{s}^{i}\right)^{2}=B_{R}
$$

where

$$
\begin{aligned}
B_{R} & =\frac{\lambda_{1}}{\lambda_{E 0}^{r}} \frac{W}{D_{i}^{r}}\left(1-e_{0}^{r} \cdot e^{i}\right) \\
& +\left[\sin 2 \gamma_{0}^{r}\left(\frac{\partial e_{0}^{r}}{\partial \gamma_{0}^{r}} \cdot e^{i}\right)+\cos ^{2} \gamma^{i}-\cos ^{2} \gamma_{0}^{r}\right]
\end{aligned}
$$

In Eq. (52), the first term always predominates. Therefore, $B_{R}>0$ is certain.

By assuming that the increment of velocity $\mathrm{d} V$ is in the plane formed by $V_{0}$ and $V_{0}^{r}$, we obtain

$$
\left.E_{r} \triangleq \frac{\mathrm{d} \gamma^{i}}{\mathrm{~d} \psi^{i}}\right|_{\tau=\tau_{0}}=\frac{n_{c}^{i}\left(\tau_{0}\right)-\cos \gamma_{0}}{n_{s}^{i}\left(\tau_{0}\right) / \cos \gamma_{0}}=\frac{a \cos \gamma_{0}}{b}
$$

where

$$
\begin{gathered}
a=\sin \gamma_{0}^{r} \cos \gamma_{0}-\cos \gamma_{0}^{r} \sin \gamma_{0} \cos \left(\psi^{r}-\psi_{0}\right) \\
b=\cos \gamma_{0}^{r} \sin \left(\psi^{r}-\psi_{0}\right)
\end{gathered}
$$


Considering $\tau=\tau_{0}$ in Eqs. (51) and (52), we let $\gamma^{i}=\gamma_{0}$, $\psi^{i}=\psi_{0}$, and $\boldsymbol{B}_{R}=\boldsymbol{B}_{R_{0}}$. Then Eqs. (51) and (53) can be solved to yield

$$
\begin{gathered}
n_{c}^{i}\left(\tau_{0}\right)=\cos \gamma_{0}+\frac{a}{\sqrt{a^{2}+b^{2}}} \sqrt{B_{R_{0}}} \\
n_{s}^{i}\left(\tau_{0}\right)=\frac{b}{\sqrt{a^{2}+b^{2}}} \sqrt{B_{R_{0}}}
\end{gathered}
$$

From Eqs. (26) and (27), we obtain the initial values of $\lambda_{\gamma 0}^{i}$ and $\lambda_{\psi 0}^{i}$. The iteration procedure is described in Appendix $B$.

In fact, Eq. (53) represents the differential equation of a great circle, which is the optimal trajectory for minimum distance from $\left(\psi_{0}, \gamma_{0}\right)$ to $\left(\psi^{r}, \gamma_{0}^{r}\right)$ on a sphere surface. It can be shown that if $B_{R}$ in Eqs. (51) and (52) is independent of $\psi^{i}$ and $\gamma^{i}$, then the great circle is also the optimal trajectory for minimum time. Since $B_{R}$ is a function of $\psi^{i}$ and $\gamma^{i}$, the minimumtime trajectory is different from the minimum-distance trajectory. However, numerical experience shows that the initial guess of $a$ and $b$ by using Eqs. (54) and (55) is so good that the error is less than 1\%. Therefore, we justify Eqs. (56) and (57) and formally let the optimal controls for simulation be

$$
\begin{aligned}
& n_{s}=n_{s}^{i}\left(\tau_{0}\right) \\
& n_{c}=n_{c}^{i}\left(\tau_{0}\right)
\end{aligned}
$$

In the preceding, we assumed that $n_{c}^{2}+n_{s}^{2}<n_{\max }^{2}$ If from Eqs. (58) and (59) we find that $n_{c}^{2}+n_{s}^{2} \geq n_{\max }^{2}$, then we let

$$
n_{c}^{2}+n_{s}^{2}=n_{\max }^{2}
$$

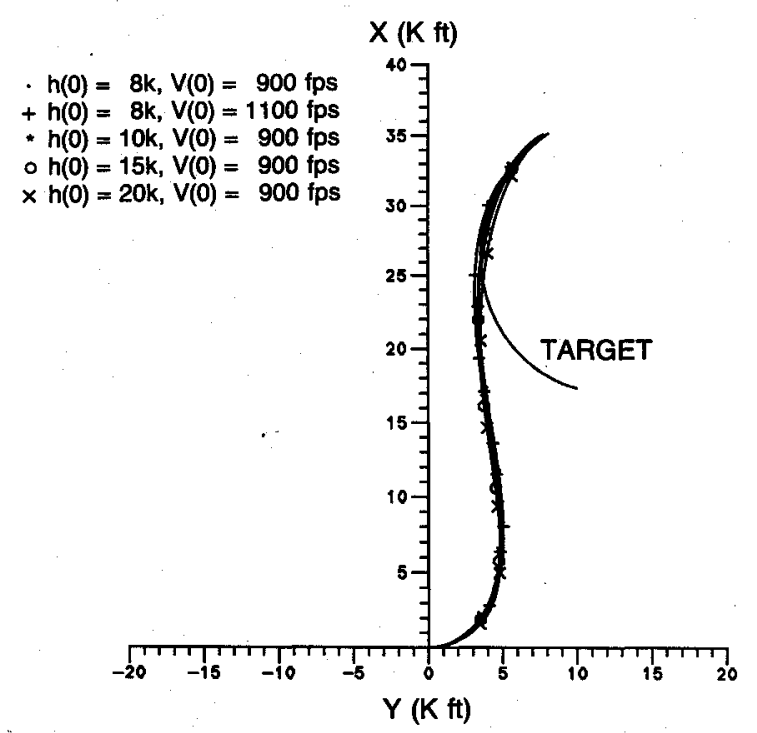

Fig. 2 Horizontal projections of trajectories.

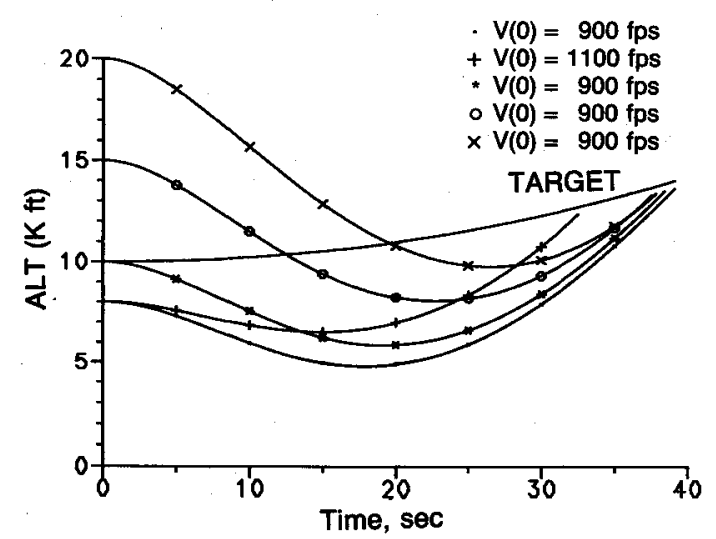

Fig. 3 Altitude profiles.
Equations (53) and (60) are used in solving for $n_{c}$ and $n_{s}$. However, Eqs. (26) and (27) can no longer be used in solving for $\lambda_{\gamma}^{i}$ and $\lambda_{\psi}^{i}$. They should be modified to include a Lagrange multiplier $\mu$.

\section{v. Higher-Order Singular Perturbation Analysis}

In the last section, we presented the zeroth-order analysis. A natural question to ask is if we can extend the analysis to a higher order with a recursive algorithm, which can be easily implemented. The answer is yes. To achieve this, we smooth out the two conflicting results in the zeroth-order approximation. They are as follows:

1) The changing rate of flight-path angle $\dot{\gamma}^{\circ}$ does not vanish identically as assumed in the zeroth-order out layer solution.

2) The flight-path and azimuth angles cannot jump instantly from their initial values to those values obtained from the outer-layer analysis without a finite time interval. This means $\tau_{f} \neq 0$ in the inner-layer solution.

To improve on such an approximation, we may let $\epsilon \dot{\gamma}_{(k)}^{o}=\dot{\gamma}_{(k-1)}^{o}$ in the $k$ th-order $(k \geq 1)$ outer-layer solution and match $x, y, z, E$ and their adjoint variables at the time $\tau_{f(k-1)}$ instead of $\tau_{0}$ in the $k$ th-order inner-layer solution. Then we may solve the problem recursively following the procedure described in the Sec. IV with little modification.

\section{Numerical Results}

The parameters of the interceptor and atmospheric data for demonstration are taken from Ref. 16. For convenience of reference, they are listed in Table 1.

Several cases are studied and the results are shown in Figs. $2-5$. For all these cases, it is assumed that initially the interceptor flies with $\gamma_{0}=0 \mathrm{deg}$ and $\psi_{0}=90 \mathrm{deg}$, and the target flies with $V_{T 0}=500 \mathrm{fps}, \gamma_{T 0}=0 \mathrm{deg}, \psi_{T 0}=-75 \mathrm{deg}, \dot{V}_{T}=4 \mathrm{ft} / \mathrm{s}^{2}$, $\dot{\gamma}_{T}=0.5 \mathrm{deg} / \mathrm{s}$, and $\dot{\psi}_{T}=g /\left(V_{T} \cos \gamma_{T}\right)$. Since the interceptor does not previously know how the target will fly in the next second, the target's future velocity is assumed to be frozen at the instant of time when the optimal controls are computed. The throttle control $\eta^{r}=1$ is employed for simulation since $\lambda_{E}^{r}<0$ is not violated throughout the whole period of interception. The horizontal projections and the altitude profiles of the trajectories are shown in Figs. 2 and 3, respectively. The difference among the cases studied concerns the initial speed and altitude of the interceptor.

An interesting result found from Fig. 3 is that the interceptor must first dive downward and then pull up to intercept an upward flight target. This result is consistent with those shown by Do Khac and Huynh. ${ }^{12}$ An explanation is that the maximum thrust and the drag are modeled as functions of the Mach number and the altitude. The higher the Mach number and the lower the altitude, the higher are the maximum thrust and the drag. When the interceptor first dives downward, it gains its speed and increases the thrust and the drag. Since the increment of the thrust is larger than that of the drag, the net gain

\begin{tabular}{|c|c|c|}
\hline \multicolumn{3}{|c|}{ and aerodynamic data } \\
\hline & $W=18,000 \mathrm{lb}$ & $0 \mathrm{ft}^{2}$ \\
\hline \multirow{4}{*}{$C_{D_{0}}=$} & 0.02 & $M<0.93$ \\
\hline & $0.02+0.2(M-0.93)$ & $0.93 \leq M<1.03$ \\
\hline & $0.04+0.06(M-1.03)$ & $1.03 \leq M<1.10$ \\
\hline & $(0.0442-0.007(M-1.10)$ & $1.10 \leq M$ \\
\hline \multirow{2}{*}{$K=$} & $\int 0.2$ & $M<1.15$ \\
\hline & $0.2+0.246(M-1.15)$ & $1.15 \leq M$ \\
\hline \multicolumn{3}{|c|}{$T_{\max }=12888\left(1+0.6 M^{2}\right) \exp (-g h / R T) \mathrm{lb}$} \\
\hline
\end{tabular}
of speed is positive. However, the interceptor cannot always dive downward and intercept an upward flight target. There-

Table 1 Parameters of interceptor and aerodynamic data 


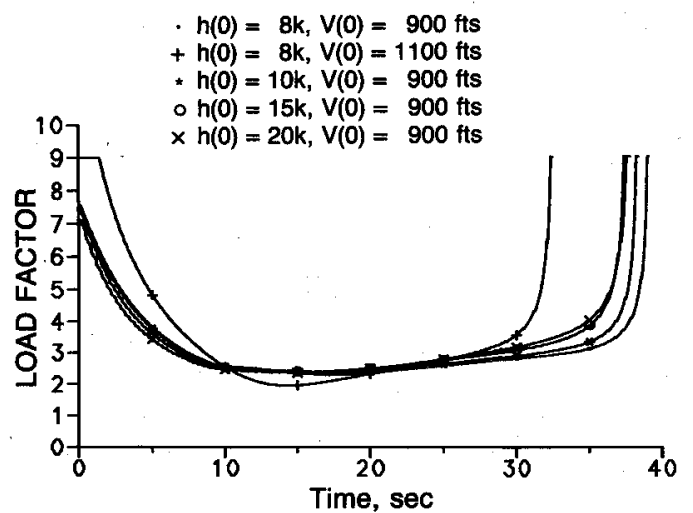

Fig. 4 Load-factor profiles.

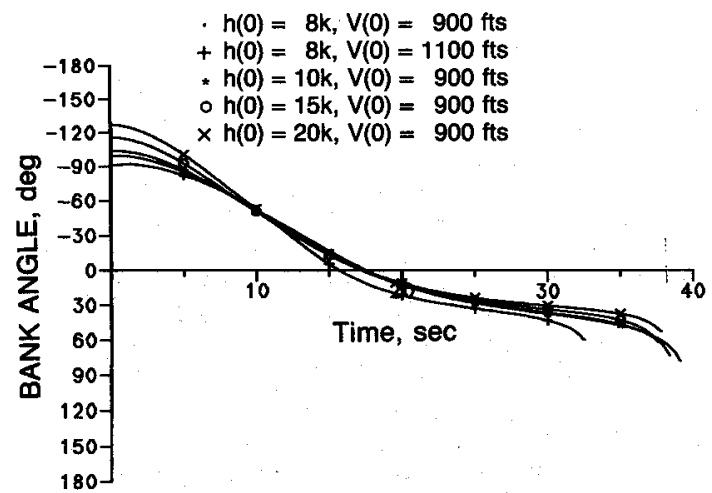

Fig. 5 Bank-angle profiles.

fore, it must pull up at a certain point and the resulting altitude profiles look like those shown in Fig. 3.

To understand the characteristics of the altitude profiles better, various combinations of the interceptor's initial altitudes and velocities are examined. Consider that in Fig. 3 the initial altitude of the interceptor is fixed at $8000 \mathrm{ft}$. Using the case with the initial speed of $900 \mathrm{fps}$ as a baseline, we find that if the initial speed is larger, say $1100 \mathrm{fps}$, the interceptor is not required to dive downward so much to intercept the target. It can be inferred that if the initial speed of the interceptor is at its maximum, then it is not necessary for the interceptor to initially dive downward to increase speed. Having this idea in mind, we examine several cases with different initial altitudes. As shown in Fig. 3, we find that if the initial velocity of an interceptor is fixed, then the interception time changes only very little with the variation of its initial altitude. For the case of high initial speed the interception time is $32.5 \mathrm{~s}$, and for all of the other cases it is about $38 \mathrm{~s}$.

Basically, to intercept a target as quickly as possible, the interceptor should turn and accelerate quickly. However, to turn quickly, the interceptor has to bank a large angle. To maintain its altitude, the interceptor has to fly with a high angle of attack, which induces a large drag. Since a large drag decelerates the interceptor, we have to compromise the bank angle with the drag. For all the cases studied in Figs. 2 and 3, their corresponding load-factor and bank-angle profiles are shown in Figs. 4 and 5, respectively. From Fig. 4, we find that if the initial speed is larger, the initial load factor can also be larger. All load-factor profiles begin with some large values and decrease to certain values, which are nearly equal to $\cos \gamma+V \dot{\gamma} / g$. To maintain high speed, the interceptor resists an early pull up, preferring to wait until near the final time to satisfy the condition $d_{f}=500 \mathrm{ft}$. Therefore, the load-factor profiles remain nearly constant for a finite period and increase suddenly when the interceptor approaches the target. In the horizontal plane, we find from Figs. 2 and 5 that the interceptor initially has negative bank angle and turns to the left. Later, the bank angle becomes positive and the interceptor turns to the right. The horizontal projections of the trajecto- ries do not change very much with the variation of the velocity or altitude. Since a large part of the lift has been used to pull the interceptor up, there remains not much to follow the turning target. Therefore, the bank angle cannot vanish at the final time as in the case of intercepting a rectilinear flight target.

To understand the singular perturbation method further, an extension to higher-order analysis has been conducted. To perform a first-order analysis, the computation time doubles. Since more computations are involved in the first-order analysis, the load-factor profiles obtained are further corrupted by noise. For the case of intercepting a rectilinear flight target with an initial speed of $900 \mathrm{fps}$, the interception time is $26.05 \mathrm{~s}$ as computed with the first-order analysis in contrast to $26.35 \mathrm{~s}$ as computed with the zeroth-order analysis. There is a $1 \%$ improvement in this case. Due to the noisy feature of its load-factor profile and a large amount of computations, the higher-order analysis is not very promising for practical implementation. However, it does serve to justify the zeroth-order analysis on a theoretical basis.

\section{Conclusions}

It has been shown that it is possible to consider only the flight-path angle and the azimuth angle as fast variables in contrast to other time-scale separations proposed in the published literatures. Physically, this time-scale separation is reasonable and its validity is not limited to the case in which the flight speed of the interceptor is near its maximum and the range of interception is very long. The performance of the controls obtained from the zeroth-order approximation appears to be very satisfactory since the higher-order analysis conducted provides little improvement.

The approach presented in this paper significantly reduces the computations involved in solving the optimal trajectory problems considered. It is observed that optimal trajectory problems of sampled-data type, such as the one presented in this paper, can always be solved with satisfactory results by using only the zeroth-order singular perturbation approach. However, special care should be made for extensions to other performance indices and other forms of terminal constraints. For the problems in which the performance indices are design parameters of vehicles and the terminal constraints are explicitly prescribed, the sampled-data type of approach may not be very efficient and sometimes not meaningful. The approach presented in this paper should then be modified to include higher-order analysis in order to obtain a satisfactory matching between the outer- and the inner-layer solutions.

\section{Appendix A: Reduced-Order Solution}

In Eq. (30), we let $\epsilon=0$ and obtain

$$
\begin{gathered}
n_{c}^{r}=\cos \gamma^{r} \\
n_{s}^{r}=0
\end{gathered}
$$

Similarly, by setting $\epsilon=0$ in Eq. (32), we obtain

$$
\begin{gathered}
\lambda_{x}^{r} \sin \gamma^{r} \cos \psi^{r}+\lambda_{y}^{r} \sin \gamma^{r} \sin \psi^{r}+\lambda_{z}^{r} \cos \gamma^{r} \\
-\lambda_{E}^{r} \frac{D_{i}^{r} \sin 2 \gamma^{r}}{W}=0 \\
\lambda_{x}^{r} \sin \psi^{r}-\lambda_{y}^{r} \cos \psi^{r}=0
\end{gathered}
$$

where we have eliminated $\lambda_{\gamma}^{r}$ by using Eq. (26). From Eqs. (19) and (20), we know that $\lambda_{x}^{r}$ and $\lambda_{y}^{r}$ are constant. Therefore, $\psi^{r}$ must be constant from Eq. (A4). Also, from Eqs. (27) and (A2), we conclude that

$$
\lambda_{\psi}^{r}=0
$$


To simplify the computation, let us define two integrals $I_{c}$ and $I_{s}$ as

$$
\begin{aligned}
& I_{c}=\int_{t_{f}^{r}}^{t_{0}} V^{r} \cos \gamma^{r} \mathrm{~d} t \\
& I_{s}=\int_{t_{f}^{r}}^{t_{0}} V^{r} \sin \gamma^{r} \mathrm{~d} t
\end{aligned}
$$

From Eqs. (29) and (31), three more differential equations are

$$
\dot{E}^{r}=\frac{\left(\eta^{r} T_{\max }-D_{0}^{r}-D_{i}^{r} \cos ^{2} \gamma^{r}\right) V^{r}}{W}
$$

$$
\begin{aligned}
\dot{\lambda}_{z}^{r}= & \lambda_{H} V_{h}^{r} \cos \gamma^{r}-\lambda_{z}^{r} V_{h}^{r} \sin \gamma^{r} \\
+ & \lambda_{E}^{r} \frac{\partial}{\partial h^{r}}\left[\frac{\left(\eta^{r} T_{\max }-D_{0}^{r}-D_{i}^{r} \cos ^{2} \gamma^{r}\right) V^{r}}{W}\right] \\
\dot{\lambda}_{E}^{r}= & -\lambda_{H} V_{E}^{r} \cos \gamma^{r}+\lambda_{z}^{r} V_{E}^{r} \sin \gamma^{r} \\
& -\lambda_{E}^{r} \frac{\partial}{\partial E^{r}}\left[\frac{\left(\eta^{r} T_{\max }-D_{0}^{r}-D_{i}^{r} \cos ^{2} \gamma^{r}\right) V^{r}}{W}\right]
\end{aligned}
$$

where

$$
\lambda_{H} \triangleq \lambda_{x}^{r} \cos \psi^{r}+\lambda_{y}^{r} \sin \psi^{r}
$$

From Eq. (A4), we find $\partial \lambda_{H} / \partial \psi^{r}=0$, i.e., $\lambda_{H}$ is independent of $\psi^{r}$. It seems that we can use the condition $H^{r}=0$ to eliminate one adjoint variable $\lambda_{z}^{r}$ or $\lambda_{E}^{r}$. This, however, entails more complicated algebraic manipulations and makes numerical errors bias.

Let $\gamma_{f}^{r}, \psi^{r}, t_{f}^{r}$, and $E_{f}^{r}$ be estimated from Eqs. (38-42) and then note that at $t=t_{f}^{r}$, the reduced-order solution satisfies

$$
H^{r}=1+\Lambda_{f} \cdot\left(V_{f}^{r} e_{f}^{r}-V_{T}\right)=0
$$

By letting $\lambda_{E f}^{r}=0$, we obtain from Eqs. (A3) and (A4),

$$
\begin{aligned}
& \boldsymbol{\Lambda}_{f} \cdot \frac{\partial e_{f}^{r}}{\partial \gamma_{f}^{r}}=0 \\
& \Lambda_{f} \cdot \frac{\partial e_{f}^{r}}{\partial \psi^{r}}=0
\end{aligned}
$$

where

$$
\begin{gathered}
\boldsymbol{\Lambda}_{f} \triangleq \lambda_{x}^{r} i+\lambda_{y}^{r} j+\lambda_{z f}^{r} \boldsymbol{k} \\
\boldsymbol{e}_{f}^{r}=\cos \gamma_{f}^{r} \cos \psi^{r} i+\cos \gamma_{f}^{r} \sin \psi^{r} j-\sin \gamma_{f}^{r} k
\end{gathered}
$$

Since $e_{f}^{r}, \partial e_{f}^{r} / \partial \gamma_{f}^{r}$, and $\partial e_{f}^{r} / \partial \psi^{r}$ are three mutually orthogonal unit vectors, from Eqs. (A13a) and (A13b), we know that $\Lambda_{f}$ is orthogonal to both $\partial e_{f}^{r} / \partial \gamma_{f}^{r}$ and $\partial e_{f}^{r} / \partial \psi^{r}$. Therefore, we conclude that

$$
\Lambda_{f}=\lambda_{r} e_{f}^{r}
$$

Substituting Eq. (A16) into Eq. (A12), we obtain

$$
\lambda_{f}=-\frac{1}{V_{f}^{r}-V_{T} \cdot e_{f}^{r}}
$$

Therefore, from Eqs. (A14) and (A16), we obtain

$$
\begin{aligned}
\lambda_{x}^{r} & =\lambda_{f} \cos \gamma_{f}^{r} \cos \psi^{r} \\
\lambda_{y}^{r} & =\lambda_{f} \cos \gamma_{f}^{r} \sin \psi^{r} \\
\lambda_{z f}^{r} & =-\lambda_{f} \sin \gamma_{f}^{r}
\end{aligned}
$$

Also, from Eq. (A11) we obtain

$$
\lambda_{H}=\lambda_{f} \cos \gamma_{f}^{r}
$$

Using the guessed quantities $\gamma_{f}^{r}$ and $\psi^{r}$, we determine the final values of the adjoint variables from Eqs. (A18-A21). In this problem, $\eta^{r}$ and $\gamma^{r}$ are the control variables. Assuming $\eta^{r}=1$, we find from the backward integration $\lambda_{E}^{r}<0$ for $t_{f}^{r}>t>t_{0}$. Therefore, from Eq. (25) it is certain that $\eta^{r}=1$. The flight-path angle $\gamma^{r}$ can be evaluated by the Newton-Raphson's method from the optimality condition in Eq. (A3). Typically, two to four integrations are adequate. Having estimated the final values and control variables, we integrate Eqs. (A6-A10) backward from $t_{f}^{r}$ to $t_{0}$. Then we obtain $I_{c}, I_{s}, E_{0}^{r}$, $\lambda_{z 0}^{r}$, and $\lambda_{E 0}^{r}$. To match the initial conditions, we define four quantities $g_{k}(k=1,2,3,4)$ by

$$
\begin{aligned}
& g_{1}=x_{0}+\left(-I_{c}+d_{f} \cos \gamma_{f}^{r}\right) \cos \psi^{r}-u_{T}\left(t_{f}^{r}-t_{0}\right) \\
& g_{2}=y_{0}+\left(-I_{c}+d_{f} \cos \gamma_{f}^{r}\right) \sin \psi^{r}-v_{T}\left(t_{f}^{r}-t_{0}\right) \\
& g_{3}=z_{0}-\left(-I_{s}+d_{f} \sin \gamma_{f}^{r}\right)-w_{T}\left(t_{f}^{r}-t_{0}\right) \\
& g_{4}=E_{0}-E_{0}^{r}
\end{aligned}
$$

Indeed, if these four quantities vanish, then we have already obtained the solution for the reduced-order problem. However, they do not vanish in general. Although rigorous approaches such as the gradient method exist, they generally require analysis to first order. This entails much more algebraic and numerical work. Instead, we choose to approximately estimate the increments $\Delta \gamma_{f}^{r}, \Delta \psi^{r}, \Delta t_{f}^{r}$, and $\Delta E_{f}^{r}$ to improve the solutions.

Using Taylor series expansion up to the second order for $I_{c}$ and $I_{s}$ and up to the first order for $E_{0}^{r}$, we can approximately evaluate the variations of $g_{k}$ by

$$
\begin{aligned}
& \Delta g_{1}=-\left(\Delta I_{c}+d_{f} \sin \gamma_{f}^{r} \Delta \gamma_{f}^{r}\right) \cos \psi^{r} \\
& +\left(I_{c}-d_{f} \cos \gamma_{f}^{r}\right) \sin \psi^{r} \Delta \psi^{r}-u_{T} \Delta t_{f}^{r} \\
& \Delta g_{2}=-\left(\Delta I_{c}+d_{f} \sin \gamma_{f}^{r} \Delta \gamma_{f}^{r}\right) \sin \psi^{r} \\
& -\left(I_{c}-d_{f} \cos \gamma_{f}^{r}\right) \cos \psi^{r} \Delta \psi^{r}-v_{T} \Delta t_{f}^{r} \\
& \quad \Delta g_{3}=\Delta I_{s}-d_{f} \cos \gamma_{f}^{r} \Delta \gamma_{f}^{r}-w_{T} \Delta t_{f}^{r} \\
& \Delta g_{4}=\left.\frac{\left(T^{r}-D_{0}^{r}-D_{i}^{r} \cos ^{2} \gamma_{f}^{r}\right) V^{r}}{W}\right|_{\left(h_{f}^{r}, V_{f}^{r}\right)} \Delta t_{f}^{r}-\Delta E_{f}^{r}
\end{aligned}
$$

where $\Delta I_{c}$ and $\Delta I_{s}$ are approximately evaluated by

$$
\begin{gathered}
-\Delta I_{c}=\Delta\left[V_{f}^{r}\left(t_{f}^{r}-t_{0}\right)-1 / 2 \dot{V}_{f}^{r}\left(t_{f}^{r}-t_{0}\right)^{2}\right] \cos \gamma_{f}^{r} \\
-\left[V_{f}^{r}\left(t_{f}^{r}-t_{0}\right)-1 / 2 \dot{V}_{f}^{r}\left(t_{f}^{r}-t_{0}\right)^{2}\right] \Delta \gamma_{f}^{r} \sin \gamma_{f}^{r} \\
-\Delta I_{s}=\Delta\left[V_{f}^{r}\left(t_{f}^{r}-t_{0}\right)-1 / 2 \dot{V}_{f}^{r}\left(t_{f}^{r}-t_{0}\right)^{2}\right] \sin \gamma_{f}^{r} \\
+\left[V_{f}^{r}\left(t_{f}^{r}-t_{0}\right)-1 / 2 \dot{V}_{f}^{r}\left(t_{f}^{r}-t_{0}\right)^{2}\right] \Delta \gamma_{f}^{r} \cos \gamma_{f}^{r}
\end{gathered}
$$

The variation $\Delta g_{k}(k=1, \cdots, 4)$ can be chosen as proportional to $-g_{k}$, i.e.,

$$
\Delta g_{k}=-\eta_{k} g_{k} \quad(k=1, \cdots, 4)
$$

where $\eta_{k}$ are constant and can be chosen as $0<\eta_{k}<1$. Typically, $\eta_{k}=0.5$ or 0.6 works very well. Relating $\Delta V_{f}^{r}$ to $\Delta E_{f}^{r}$, we can solve Eqs. (A26-A29) simultaneously for $\Delta t_{f}^{r}, \Delta \gamma_{f}^{r}, \Delta E_{f}^{r}$, and $\Delta \psi^{r}$. Then we renew the guessed quantities by

$$
\begin{gathered}
t_{f \text { (new) }}^{r}=t_{f(\text { old })}^{r}+\Delta t_{f}^{r} \\
\gamma_{f \text { (new) }}^{r}=\gamma_{f \text { (old) }}^{r}+\Delta \gamma_{f}^{r}
\end{gathered}
$$




$$
\begin{aligned}
& E_{f \text { (new) }}^{r}=E_{f(\text { old })}^{r}+\Delta E_{f}^{r} \\
& \psi_{\text {(new) }}^{r}=\psi_{\text {(old) }}^{r}+\Delta \psi^{r}
\end{aligned}
$$

and a new cycle of iteration can be started. Typically, the solution will converge with better than $95 \%$ accuracy in four to six iterations.

\section{Appendix B: Zeroth-Order Inner-Layer Solution}

Setting $\epsilon=0$ in Eq. (33), we find that $x^{i}, y^{i}, z^{i}$, and $E^{i}$ are constant and equal to their initial values, respectively. Similarly, setting $\epsilon=0$ in Eq. (35), we find that $\lambda_{x}^{i}, \lambda_{y}^{i}, \lambda_{z}^{i}$, and $\lambda_{E}^{i}$ are constant. From the matching conditions, $\lambda_{x}^{i}=\lambda_{x}^{r}, \lambda_{y}^{i}=\lambda_{y}^{r}$, $\lambda_{z}^{i}=\lambda_{z 0}^{r}$, and $\lambda_{E}^{i}=\lambda_{E 0}^{r}$. The four differential equations that need to be integrated are

$$
\begin{array}{cc}
\frac{\mathrm{d} \gamma^{i}}{\mathrm{~d} \tau}=\frac{g}{V_{0}^{r}}\left(n_{c}^{i}-\cos \gamma^{i}\right), & \gamma^{i}\left(\tau_{0}\right)=\gamma_{0} \\
\frac{\mathrm{d} \psi^{i}}{\mathrm{~d} \tau}=\frac{g}{V_{0}^{r}} \frac{n_{s}^{i}}{\cos \gamma^{i}}, & \psi^{i}\left(\tau_{0}\right)=\psi_{0} \\
\frac{\mathrm{d} \lambda_{\gamma}^{i}}{\mathrm{~d} \tau}=-\Lambda_{0} \cdot V_{0}^{r} \frac{\partial e^{i}}{\partial \gamma^{i}}-\lambda_{\gamma}^{i} \frac{g}{V_{0}^{r}} \sin \gamma^{i}-\lambda_{\psi}^{i} \frac{g}{V_{0}^{r}} \frac{n_{s}^{i} \sin \gamma^{i}}{\cos ^{2} \gamma^{i}} & \frac{\mathrm{d} \lambda_{\psi}^{i}}{\mathrm{~d} \tau}=-\Lambda_{0} \cdot V_{0}^{r} \frac{\partial e^{i}}{\partial \psi^{i}}
\end{array}
$$

Following the description in Sec. IV, we can guess the initial values $\lambda_{\gamma}^{i}\left(\tau_{0}\right)$ and $\lambda_{\psi}^{i}\left(\tau_{0}\right)$. The control variables $n_{c}^{i}$ and $n_{s}^{i}$ can be evaluated by

$$
\begin{gathered}
n_{c}^{i}=\frac{\lambda_{\gamma}^{i} \frac{g}{V_{0}^{r}}}{2 \lambda_{E 0}^{r} V_{0}^{r} \frac{D_{i}^{r}}{W}} \\
n_{s}^{i}=\frac{\lambda_{\psi}^{i} \frac{g}{V_{0}^{r}} \frac{1}{\cos \gamma^{i}}}{2 \lambda_{E 0}^{r} V_{0}^{r} \frac{D_{i}^{r}}{W}}
\end{gathered}
$$

if $\left(n_{c}^{i}\right)^{2}+\left(n_{s}^{i}\right)^{2} \leq n_{\max }^{2}$. Otherwise, some modification should be made. Having obtained the initial conditions and control variables, we can then integrate Eqs. (B1-B4) until

$$
a_{i r} \dot{a}_{i r}+b_{i r} \dot{b}_{i r}=0
$$

where

$$
\begin{gathered}
a_{i r}=\sin \gamma_{0}^{r} \cos \gamma^{i}-\cos \gamma_{0}^{r} \sin \gamma^{i} \cos \left(\psi^{r}-\psi^{i}\right) \\
b_{i r}=\cos \gamma_{0}^{r} \sin \left(\psi^{r}-\psi^{i}\right)
\end{gathered}
$$

Indeed, if $a_{i r}=0$ and $b_{i r}=0$, then we already have the correct solution. But in general it is not so. We then modify the guessed values $a$ and $b$ by

$$
\begin{aligned}
& a_{\text {(new) }}=a_{\text {(old) }}+\sigma_{E}\left[a_{\text {(old) }}-a_{0 f}\right] \\
& b_{\text {(new) }}=b_{\text {(old) }}+\sigma_{E}\left[b_{\text {(old) }}-b_{0 f}\right]
\end{aligned}
$$

where

$$
\begin{gathered}
a_{0 f}=\sin \gamma_{f}^{i} \cos \gamma_{0}-\cos \gamma_{f}^{i} \sin \gamma_{0} \cos \left(\psi_{f}^{i}-\psi_{0}\right) \\
b_{0 f}=\cos \gamma_{f}^{i} \sin \left(\psi_{f}^{i}-\psi_{0}\right)
\end{gathered}
$$

and $\sigma_{E}$ can be chosen so that $0<\sigma_{E}<1$. A typical value for $\sigma_{E}$ is 0.25 .

After obtaining the modified ratio $a$ and $b$, we can start a new cycle of iteration. Certainly this is not necessary, since the error in the initial guess using Eq. (53) is generally less than $1 \%$.

\section{Acknowledgment}

This work was supported in part by the U.S. Army Strategic Defense Command under Contract DASG 60-88-C-0037.

\section{References}

${ }^{1}$ Calise, A. J., "Singular Perturbation Methods for Variational Problems in Aircraft Flight," IEEE Transactions on Automatic Control, Vol. AC-21, No. 3, 1976, pp. 345-353.

${ }^{2}$ Ardema, M. D., "Solution of the Minimum Time-to-Climb Problem by Matched Asymptotic Expansions," AIAA Journal, Vol. 14, No. 7, 1976, pp. 843-850.

${ }^{3}$ Shridhar, B., and Gupta, N. K., "Missile Guidance Laws Based on Singular Perturbation Methodology," Journal of Guidance and Control, Vol. 3, No. 2, 1980, pp. 158-165.

${ }^{4}$ Visser, H. G., and Shinar, J., "A Highly Accurate Feedback Approximation for Horizontal Variable-Speed Interceptions," Journal of Guidance, Control, and Dynamics, Vol. 9, No. 6, 1986, pp. 691-698.

${ }^{5}$ Calise, A. J., "A New Boundary Layer Matching Procedure for Singularly Perturbed Systems," IEEE Transactions on Automatic Control, Vol. AC-23, No. 3, 1978, pp. 434-438.

${ }^{6}$ Calise, A. J., "A Singular Perturbation Analysis of Optimal Aerodynamic and Thrust Magnitude Control," IEEE Transactions on Automatic Control, Vol. AC-24, No. 5, 1979, pp. 720-730.

${ }^{7}$ Rajan, N., and Ardema, M. D., "Interception in Three Dimensions-An Energy Formulation," Proceedings of the AIAA Atmospheric Flight Mechanics Conference, AIAA, New York, 1983, pp. 1-10.

${ }^{8}$ Visser, H. G., Kelley, H. J., and Cliff, E. M., "Energy Management of Three-Dimensional Minimum-Time Intercept," Journal of Guidance, Control, and Dynamics, Vol. 10, No. 6, 1987, pp. 574-580.

${ }^{9}$ Ardema, M. D., and Rajan, N., "Separation of Time Scales in Aircraft Trajectory Optimization," Journal of Guidance, Control, and Dynamics, Vol. 8, No. 2, 1985, pp. 275-278.

${ }^{10}$ Ardema, M. D., and Yang, L., "Interior Transition Layers in Flightpath Optimization," Journal of Guidance, Control, and Dynamics, Vol. 11, No. 1, 1988, pp. 13-18.

${ }^{11}$ Calise, A. J., "Singular Perturbation Techniques for On-Line Optimal Flight-Path Control," Journal of Guidance and Control, Vol. 4, No. 4, 1981, pp. 398-405.

${ }^{12}$ Do Khac, M., and Huynh, H. T. " "Real-Time Guidance Laws for Three-Dimensional Interception," ICAS Congress, ONERA Paper TP 1988-95, Jerusalem, Sept. 1988.

${ }^{13}$ Vinh, N. X., Optimal Trajectories in Atmospheric Flight, Elsevier Scientific, Amsterdam, 1981, Chap. 3.

${ }^{14}$ Lin, D. F., "Minimum-Time Three-Dimensional Turn to a Point of Supersonic Aircraft," Journal of Guidance, Control, and Dynamics, Vol. 5, No. 5, 1982, pp. 512-520.

${ }^{15}$ Bryson, A. E., and Ho, Y. C., Applied Optimal Control, Wiley, New York, 1975

16Uehara, S., Stewart, H. J., and Wood, L. J., "Minimum-Time Loop Maneuvers of Jet Aircraft," Journal of Aircraft, Vol. 15, No. 8,1978 , pp. $449-455$. 